\title{
Antenna Placement and Performance Tradeoffs with Hand Blockage in Millimeter Wave Systems
}

\author{
Vasanthan Raghavan, Mei-Li (Clara) Chi, M. Ali Tassoudji, Ozge H. Koymen, and Junyi Li
}

\begin{abstract}
The ongoing commercial deployment of millimeter wave systems brings into focus a number of practical issues in form factor user equipment (UE) design. With wavelengths becoming smaller, antenna gain patterns becoming directional, and link budgets critically dependent on beamforming, it becomes imperative to use a number of antenna modules at different locations of the UE for good performance. While more antennas/modules can enhance beamforming array gains, it comes with the tradeoff of higher component cost, power consumption of the associated radio frequency circuitry, and a beam management overhead in learning the appropriate beam weights. Thus, the goal of a good UE design is to provide robust spherical coverage corresponding to good array gains over the entire sphere around the UE with a low beam management overhead, complexity, and cost. The scope of this paper is to study the implications of two popular commercial millimeter wave UE designs (a face and an edge design) on spherical coverage. We show that analog beam codebooks can result in good performance for both the designs, and the edge design provides a better tradeoff in terms of robust performance (with hand blockage), beam management overhead, implementation complexity from an antenna placement standpoint and cost.
\end{abstract}

Index Terms-Millimeter wave, commercial deployments, spherical coverage, antenna placement, modular design, UE design, 5G-New Radio, hand blockage.

\section{INTRODUCTION}

Enhanced spectrum availability over a part of the millimeter wave band $(\sim 24-100 \mathrm{GHz})$ has led to the focus of Fifth Generation $(5 \mathrm{G})$ wireless systems at these bands to meet the increased data rate and low latency requirements [1]-[4]. With the ongoing standardization and testing of such systems, a number of practical issues have to be solved before (and through) commercial deployments. In this context, it is now well-understood that millimeter wave link margins are sufficient to allow small-to-medium cell coverage by leveraging the increased beamforming array gains possible with the use of a larger number of antennas within the same physical aperture [5]-[12]. In contrast to sub-6 GHz systems, such array gains are constrained by the fact that an antenna at millimeter wave carrier frequencies is inherently directional 1 . As with legacy systems, the base-station design

The authors are with Qualcomm Flarion Technologies, Bridgewater, NJ 08807, USA and Qualcomm Corporate R\&D, San Diego, CA 92121, USA. Email: vasanthan_raghavan@ieee.org, clarachi@gmail.com, \{alit, okoymen, junyil\}@qti.qualcomm.com.

${ }^{1}$ In particular, a millimeter wave antenna can produce meaningful gains only over a certain spatial coverage region (typically a $120^{\circ} \times 120^{\circ}$ region of the sphere). can naturally incorporate this constraint by focussing on realizing sectoral coverage (typically a $120^{\circ}$ or $90^{\circ}$ region in the azimuth plane, with a narrow $30^{\circ}-45^{\circ}$ elevation steering/coverage). However, a similar design objective at the user equipment (UE) end can lead to significant performance degradation if useful signals cannot be picked up from different base-stations serving in different sectors (individually or in coordination) or from different clusters (within the same base-station) that correspond to widely disparate angles of arrival. Thus, a cumulative distribution function (CDF) of the spherical coverage that captures the beamforming array gain achievable with the UE's antennas in a sphere $\left(360^{\circ} \times 180^{\circ}\right.$ in azimuth and elevation, respectively) around it becomes a paramount benchmark of UE design and performance.

In particular, a good spherical coverage CDF corresponds to not only good array gains in the top few (e.g., top 30) percentile points, but also in the middle (e.g., $30^{\text {th }}-75^{\text {th }}$ ) percentile points. In particular, as we will see later in the sequel, the hand can lead to a wide spatial area blockage and good coverage cannot be ensured with any UE design over the tail (approximately bottom 25) percentile points. With only directional/modular coverage possible with millimeter wave antennas, it becomes necessary to use multi-antenna subarrays at different locations of the UE to realize a good spherical coverage CDF. Such a construction becomes even more critical to provide subarray diversity and robustness to hand blockage.

On the other hand, with a reduction in wavelength, a number of individual antenna elements can be placed/mounted at the UE side within the same form factor allowing increased array gains that is hitherto not possible at sub$6 \mathrm{GHz}$. While such a possibility makes a theoretical case for packing as many antennas as possible at the UE side (contingent on competing space with antennas at sub-6 GHz frequencies, WiFi and Bluetooth systems, cameras, sensors, and the associated circuit elements, etc.), the added cost of millimeter wave antenna modules and associated radio frequency (RF) front end circuitry (e.g., power and low-noise amplifiers, mixers, etc.) and the concomitant power increase puts a practical limit on how many antennas can be gainfully employed in a millimeter wave UE. More importantly, while the use of a large number of antennas (and antenna modules) can theoretically lead to increased beamforming gains, if these capabilities are not practically exercisable with a low beam management overhead, the capabilities can quickly 
turn out to be onerous and a curse rather than a blessing.

With form factor/real-estate considerations at the UE side, the focus of this work is on the practical realm: antenna placement and the impact of different UE designs on spherical coverage, both with and without hand blockage. To understand the tradeoffs in terms of antenna placement, we consider two popular UE designs in this work. These are: i) a face design that has antenna modules, with planar dualpolarized patch subarrays and linear dipole subarrays on the edges, placed on the front and back faces of the UE and ii) an edge design with linear dual-polarized patch subarrays placed on three edges of the UE. These designs have been introduced/studied as possible commercial UE designs by different original equipment manufacturers (OEMs) for compliance and testing studies of millimeter wave systems at the Third Generation Partnership Project (3GPP) Working Group 4 (WG4) meetings; see e.g., [13]-[25], etc.

Due to the complicated impact of different substrate materials in the UE on the antenna response function, such studies cannot be conducted theoretically. Thus, for both the UE designs, the individual antenna element response functions in both polarizations are obtained over the sphere using the Ansys High-Frequency antenna response/Structure Simulator (HFSS) commercial software suite [26]. With these individual response functions, array gains corresponding to different beamforming schemes such as maximum ratio combining (MRC), equal gain combining (EGC), an RF/analog beam codebook-based solution, and antenna selection are studied. The MRC and EGC schemes can be viewed as optimistic upper bounds (with different beam weight constraints) on beamforming performance given a certain antenna/module capability. The antenna selection scheme can be viewed as a pessimistic lower bound corresponding to legacy system design. It can also be viewed as the performance obtained after the initial acquisition phase in 3GPP 5G-New Radio (NR) using 2 the P-1 procedure [27] with a single antenna exciting pseudo-omni beam. On the other hand, the RF/analog beam codebook scheme is a $3 \mathrm{GPP} 5 \mathrm{G}-\mathrm{NR}$-compatible scheme for realizing practical beamforming performance in millimeter wave systems at the end of the $\mathrm{P}-1 / 2 / 3$ procedures. The size of the beamforming codebook determines the tradeoff between beam management overhead and gap to optimal MRC/EGC performance. The larger the codebook size, the higher the beam management overhead and smaller the gap to optimal performance (and vice versa).

We consider both Portrait and Landscape mode blockages with blockage model from the $3 \mathrm{GPP}$ specification in TR 38.901 [5] as well as more realistic models from measurements with a $28 \mathrm{GHz}$ experimental prototype [28], [29].

\footnotetext{
${ }^{2}$ In 3GPP TR 38.912 [27], hierarchical beamforming at the base-station and UE ends are proposed in three phases: an initial acquisition or P-1 phase of wide beams at both ends, and beam refinement (or P-2 and P-3 phases) at the base-station and UE ends, respectively. The Release $15 \mathrm{spec}$ of 5G-NR essentially follows these outlines without any explicit citations to $\mathrm{P}-1 / 2 / 3$ procedures.
}

Based on these studies, the main conclusions of this work are as follows.

- Good RF/analog beam codebooks that tradeoff beam management overhead for robust performance over the sphere can be designed for both the UE designs considered in this work. These codebooks can realize a significant fraction (within 1-2 dB) of the optimal array gains possible for these designs. Substantial performance improvement (from 2-6 dB depending on the size of the subarrays and the precise direction of the cluster/path) is seen with the codebook scheme over the antenna selection scheme. This performance improvement captures the benefit of performing the P3 beam refinement at the UE side over the P-1 initial acquisition phase.

- The flat $30 \mathrm{~dB}$ loss over the blocked spatial region assumed with the 3GPP blockage model in [5] leads to an abrupt and dramatic loss in performance over these regions in both Portrait and Landscape modes. A more smoother performance degradation is seen with the model proposed in [29]. Nevertheless, blockage is seen to produce a bimodal behavior of nearly unobstructed transmission/reception over the unblocked region, and unrecoverable signal over the blocked region. This bimodal performance reinforces the criticality of subarray/modular diversity, channel richness, and alternate viable cluster/path learning for switching to a potentially alternate cluster/path in the case of an impending blockage of a serving cluster/path [29], [30].

- While the use of an increased number of antenna modules would suggest a better robustness to blockage, the learning overhead associated with codebook-based beam training suggests a good tradeoff point in terms of the number of antenna modules at an intermediate value. The face and edge designs are generally competitive with each other with no strong advantages in performance for either design. In general, practical advantages can be seen with a smaller number of subarrays/modules to be learned/trained.

- That said, the face design has a strong implementationlevel complexity arising from the need to find realestate on the front face of the UE (something that is typically reserved for almost bezel-less displays in current and next generations of UEs). Further, placing the antenna module underneath a glass/plastic display can lead to additional signal deterioration [10], [31], [32] that is unaccounted for. A third complexity associated with the face design are transmissions that could cause a major exposure in the direction of unintended body parts (e.g., eye, skin, etc.). Thus, the edge design provides a better tradeoff in terms of robust performance, beam management overhead, implementation complexity, and cost, suggesting its utility in commercial millimeter wave UE designs. 
Novelty of this work: The novelty of this paper relative to prior works on beamforming is now explained. While there are a number of works on beamforming for millimeter wave systems (single- and multi-cell aspects), a system level study of the tradeoffs in antenna placement in a form factor constrained UE have not been explored prior to this work. Quite simply, unlike sub-6 $\mathrm{GHz}$ systems where antenna placement does not matter much in terms of system level performance, the success of millimeter wave systems is critically dependent on good antenna placement and there are no fair studies of different UE designs in the literature. Specifically, notions such as spherical coverage have not been studied in an academic context to compare multiple UE designs, either with or without hand blockage. Such studies are important given the impending commercialization of millimeter wave systems [33] and the need for the robustness of such designs with hand blockage. This work focusses on answers to these practically-inspired problems.

In terms of the prior work of the authors, [10] compares the macroscopic channel features (such as path loss exponents, delay spread, penetration loss, etc.) via measurements in different deployment scenarios across different carrier frequencies, but limits itself to channel modeling and its implications. The work in [28] describes a $28 \mathrm{GHz}$ experimental prototype with a proprietary pre-5G subframe structure and the prototype's robustness in terms of performance with indoor and outdoor mobility. The work in [29] describes the limitations of the $3 \mathrm{GPP}$ hand blockage model relative to form factor UE measurements with the hand. To overcome these limitations, it proposes a simplified alternate model describing the hand blockage loss. Both these models are used in this work to capture spherical coverage loss with hand blockage. The scope of [30] is a broad summary (for a wider reach) of the spatio-temporal impact of hand blockage in millimeter wave systems. A brief explanation of the poor fit of the 3GPP model as illustrated in [29] along with the time-scales of blockage and link disruption, UE side impact and possible mitigation strategies are considered in [30]. In [34], different types of beamforming schemes (such as those based on singular vectors, array steering vectors, compressive sensing schemes, etc.) are studied and a simple codebook-based beamforming scheme is shown to be robust, practical and scalable for initial link acquisition in millimeter wave systems. Such studies have also motivated the choice behind the agreed protocol in 3GPP TR 38.912 [27].

Organization: This paper is organized as follows. Sec. [ introduces the UE designs studied in this work, their design tradeoffs in terms of practical implementation, and connections to prior UE design work in academia as well as commercial designs. Sec. III explains the system setup such as the nature and scope of the beamforming algorithms considered, performance metric used in the study for quantifying the goodness of the designs, RF/analog beam codebooks used for these designs, and the considered blockage models.
Sec. IV presents the spherical coverage CDF tradeoffs for these designs (as well as more sophisticated designs) with the different beamforming schemes and blockage models as well as head-to-head comparisons across the designs. Sec. $\mathbf{D}$ presents some concluding remarks and possible directions for future studies.

\section{UE Designs for Spherical Coverage Studies}



Fig. 1. Pictorial illustration of the face and edge designs considered in this work along with the antenna module structure in these designs and the boresight directions of the main scanning plane(s) of all the subarrays.

\section{A. Designs Studied in This Work}

For spherical coverage studies, we consider two popular UE designs in this work as illustrated in Fig. 1] These designs are:

- A face design with two antenna modules (on the front and back corners of the UE) with each module made of $2 \times 2$ dual-polarized patch subarrays and two $2 \times 1$ (single-polarized) dipole subarrays at the edges of the module. Note that it is typical to count a dual-polarized patch antenna as two antenna elements since they are fed by two independent antenna feeds. Thus, for each antenna module in the face design, the $2 \times 2$ dualpolarized patch subarray counts for 8 antenna elements and the dipole subarrays count for 4 antenna elements leading to a total of 12 antenna elements per module. Since there are two modules, we have 24 antenna elements in all in this design.

- An edge design with three antenna modules (on three sides of the UE) with each module made of $4 \times 1$ dual-polarized patch subarrays alone. For each antenna module in the edge design, the dual-polarized patch subarray accounts for 8 antenna elements. Along with 
the use of three modules, we have 24 elements in all here.

Both these designs are tailored for dual layer transmission/reception at $28 \mathrm{GHz}$. These two layers could be mapped/connected to the two polarizations of the patch subarrays, or two paired dipole subarrays, or a patch and a dipole subarray (the latter two pairs arise only in the face design). Information on the number of antenna modules, number of antennas (in both polarizations), approximate elemental gains of the antennas, and number and description of the different subarrays in these designs are summarized in Table I. A pictorial illustration of the antenna module design and the boresight directions of the main scanning plane of all the subarrays in each design are marked with blue arrows in Fig. 1

\section{B. Design Tradeoffs and Practical Implementation Issues}

Face Design: The face design is hard to implement in practice. This is because in addition to finding sufficient realestate within the display uni 3 of the UE for the antenna module to be mounted, careful mounting of the antenna modules can also lead to manufacturing complexities and cost/time overruns. Further, such a design can incur significant additional radiation losses due to penetration of millimeter wave signals through typical display materials (e.g., glass, plastic, ceramic, etc.). In particular, works such as [10], [31] and [32] point out that the loss is material (permittivity and loss tangent)-dependent, depends on the antenna type (dipole or patch), clearance between display and cover, and can cover a wide bandwidth. The cover/display acts as a lens/dispersive medium and scatters the signal compared to the baseline case of no cover. A glass cover will scatter more energy than a plastic cover resulting in attenuation of signals in certain directions and comparable performance or even amplification of signals in certain other directions (all relative to the case with no cover).

Another issue with the use of planar arrays on the front face of the UE is exposure of sensitive body parts (e.g., eye, skin, etc.) to the beamformed signal with high energy. On the other hand, the use of planar arrays with each antenna module (instead of linear arrays) allows a twodimensional beam scanning that allows a better parsing of the clusters in the channel, as well as limiting signal leakage (or interference) in unintended directions possible with onedimensional beam scanning. Thus, a reasonable spherical coverage can be anticipated with the use of only two antenna modules (on the front and back) which can minimize cost, power consumption, as well as beam management overhead.

Specific to the face design, dipole antennas are more affected by placement issues than patch antennas. Thus, the

\footnotetext{
${ }^{3}$ Almost bezel-less displays have become popular in the current generation of UEs and will be increasingly used in future designs. This constraint renders the use of a planar array at least on the front face of the UE questionable.
}

beamforming performance with the dipole antennas in these designs can show a big deviation from expected performance in Freespace. This deviation requires a careful design of housing in these designs. Further, dipole antennas require more area (and a bigger size) than patch antennas. Thus, in thin UE designs, the antenna modules may need to be tilted or placed at an angle resulting in a more complicated spherical coverage tradeoff. On the other hand, dipoles allow a more broadband coverage relative to patch antenna elements allowing the reuse of the same antenna design across different bands/geographies [35].

Edge Design: The edge design appears to be the easiest from a practical implementation standpoint. By placing antennas on the edges, the edge design takes advantage of the robustness to the precise choice of location of the antenna modules on the edge and thus this design can minimize mounting problems. Since a commercial UE design has to accommodate different real-estate constraints associated with sensors, cameras, battery, other antennas, etc., this robustness adds a significant level of versatility to UE design. Additionally, the edge placement can significantly reduce display-related penetration losses (relative to the face design).

On the other hand, analogous to display-related losses for the face design, frame-related losses can accrue for the edge design. Note that typical frame materials include plastic and metal. The typical impact of these materials is to decrease the beam's strength and/or to tilt or steer the beams away from their intended directions. From prior works such as [31] and [32], it is known that additional losses are a function of the permittivity and loss tangent of the material, antenna type, clearance between frame and antenna substrate, beam steering direction, etc. In the case of plastic frames, commonly used in a broad range of UEs, these losses are usually minimal. However, metallic frames can lead to further losses that need to be included in our studies. To be fair to both the face and edge designs, we have not included display losses for the face design as well as frame losses for the edge design in our studies. This is done so as to give a big-picture idea of the tradeoffs involved in UE design instead of incorporating every implementation aspect in high specificity. For practical implementations, both these additional losses need to be included.

The use of linear arrays (a UE's typical form-factor only allows linear arrays on the edges) leads to the need of at least four antenna modules for full spherical coverage in Freespace resulting in increased cost, power consumption as well as beam management overhead. In general, the edge design tries to appropriate the good features of the face design such as a small number of antenna modules by adding a layer of robustness in design. By anticipating poor spherical coverage performance over one part of the sphere, the number of antenna modules can be reduced from four to three. This poor performance could be due to the edge 
TABLE I

Design Parameters for the Face and Edge Designs

\begin{tabular}{|c||c|c|}
\hline Parameter of interest & Face design & Edge design \\
\hline \hline No. of antenna modules & 2 & 3 \\
\hline No. of antenna elements & 24 & 24 \\
\hline No. of subarrays per module & $4(2 \times 2$ dual-polarized patch subarrays, & $2(4 \times 1$ dual-polarized patch subarrays $)$ \\
& $2 \times 1$ and $1 \times 2$ dipole subarrays $)$ & 4 for each patch subarray \\
\hline No. of beams per subarray & 4 for patch and 2 for dipole subarrays & $8(=4$ beams $\times 2$ patch subarrays $)$ \\
\hline Codebook size per module & $12(=4$ beams $\times 2$ patch subarrays \\
& +2 beams $\times 2$ dipole subarrays $)$ & $24=8 \times 3$ \\
\hline Total codebook size & $24=12 \times 2$ & $\sim 5.5 \mathrm{dBi}$ \\
\hline Elemental gain & $\sim 5.8$ and $\sim 4.7 \mathrm{dBi}$ for patches and dipoles & \\
\hline
\end{tabular}

pointing away from the serving base-station(s) and towards the ground plane in Portrait mode, or due to the presence of the hand in the Landscape mode. In terms of exposure, some subarrays can steer energy towards the body of the user with minor signal energy peaks. Thus, relative to the face design, the edge design is expected to have rather minor exposurerelated concerns. With this background, it is of interest in understanding the spherical coverage $\mathrm{CDF}$ performance with these UE designs.

\section{Connections to Other Designs}

The readers are pointed to [36]-[38] for some recent studies on design tradeoffs of $5 \mathrm{G}$ antenna arrays with form factor considerations. In the context of spherical coverage studies, a number of reference UE architecture designs have been introduced at 3GPP in terms of developing testing and conformance requirement specifications for the effective isotropically radiated power (EIRP) with millimeter wave transmissions. For example, the TR $38.803 \mathrm{spec}$ document has a number of potential UE reference architectures for the high bands (> $24 \mathrm{GHz}$ ) [13, Sec. 6.2.1.1, pp. 107-108]; also, see [39]. In the spherical coverage studies considered at the WG4 level, a number of companies have proposed and considered diverse UE designs. These designs include the proposals by Apple and Intel [14], Samsung, Apple and Intel [15], NTT DOCOMO [16], Qualcomm [17], [18], Sony [19]-[22], Sony and Ericsson [23], [24], LG Electronics [25], etc.

In particular, a design similar to the face design has been considered in [13], [36] as well as by [15], [16], [20], [21], [25]. The edge design has been proposed and studied in [17], [20]. More details on its practical implementation considerations (such as packaging and performance with glass/plastic display materials) are discussed in [18]. Many other designs are considered in [24], [39], [40]. Thus, these designs can be seen to be representative and reflective of popular commercial UE deployments (current as well as the near-future) in the millimeter wave regime.

Some recent work documenting the robustness advantages of large circular arrays for outdoor deployments include [41].
In this context, in terms of the directivity pattern, a linear array is more asymmetric due to the one-dimensional nature of the array relative to a planar array which is two-dimensional. Similarly, a circular array is more symmetric due to the effective two-dimensional nature of the array. On the other hand, due to form factor constraints, circular arrays can only be deployed on the front or back face of the UE instead of the edges. Thus, circular arrays share the same pros and cons as planar arrays and the face design (in particular), such as better parsing of clusters in the channel, limiting interference in unintended directions, exposure constraints, additional losses due to face material, etc.

While one can leverage the circle's isoperimetric properties (maximizing area for a given circumference) by using fewer antenna elements for the same desired directivity relative to a linear or a planar array, this advantage is striking primarily with large arrays (such as those used at the base-station end or in a customer premises equipment or for applications such as radar, automotive, etc.) [41]. For small-sized arrays such as those in a UE, the complexity of designing and deploying a circular array [42] overwhelms any potential advantages in terms of fewer antenna elements.

\section{System Setup for Spherical Coverage STUDIES}

\section{A. Beamforming Schemes}

We study the spherical coverage CDF with four beamforming schemes that co-phase multiple antenna transmission/reception in various ways in this work. To describe these schemes, given a subarray of $N$ antenna elements, let $\mathbf{E}_{\Theta}(\theta, \phi)=\left[\mathrm{E}_{\Theta, 1}(\theta, \phi), \cdots, \mathrm{E}_{\Theta, N}(\theta, \phi)\right]$ and $\mathbf{E}_{\Phi}(\theta, \phi)=$ $\left[\mathrm{E}_{\Phi, 1}(\theta, \phi), \cdots, \mathrm{E}_{\Phi, N}(\theta, \phi)\right]$ denote the antenna response functions in the $\Theta$ and $\Phi$ polarization 4 along a certain

\footnotetext{
${ }^{4}$ Typically, antenna response functions are specified in the $\Theta$ and $\Phi$ polarizations to avoid unnecessary confusion with notations such as $\mathrm{H}$ or V-polarizations that are associated with the point on the sphere where the antenna responses are computed.
} 
direction $5(\theta, \phi)$ of the sphere with $\theta$ and $\phi$ denoting the zenith/elevation and azimuth angles, respectively. As an illustration, in the scenario of an array with $\left(N_{\mathrm{x}}, N_{\mathrm{y}}, N_{\mathrm{z}}\right)$ antennas on the X-Y-Z axes where $N=N_{\mathrm{x}} N_{\mathrm{y}} N_{\mathrm{z}}$ and $\lambda / 2$ inter-antenna element spacing across all the axes, the ideal antenna response function of the $n$-th antenna is given as [43]

$$
\begin{aligned}
& \mathrm{E}_{\Theta, n}(\theta, \phi)= \mathrm{E}_{\Phi, n}(\theta, \phi)=\frac{1}{\sqrt{N}} \cdot \\
& e^{j \pi \cdot\left(n_{\times} \sin (\theta) \cos (\phi)+n_{\mathrm{y}} \sin (\theta) \sin (\phi)+n_{\mathrm{z}} \cos (\theta)\right)}, \\
& 1 \leq n \leq N
\end{aligned}
$$

where $n-1=n_{\mathrm{x}}+n_{\mathrm{y}} N_{\mathrm{x}}+n_{\mathrm{z}} N_{\mathrm{x}} N_{\mathrm{y}}$ with $0 \leq n_{\mathrm{x}} \leq N_{\mathrm{x}}-1$, $0 \leq n_{\mathrm{y}} \leq N_{\mathrm{y}}-1$ and $0 \leq n_{\mathrm{z}} \leq N_{\mathrm{z}}-1$.

The considered beamforming schemes in this paper are as follows.

- Scheme 1: The first scheme corresponds to MRC [44] in every direction $(\theta, \phi)$ of the sphere without any phase or amplitude quantization of the beamforming vector. Since infinite-precision is assumed for phase, amplitude as well as directional resolution (co-phasing beams are used in every direction), this scheme serves as an optimistic upper bound on the spherical coverage performance of the UE design. In particular, the MRC scheme over the $\mathrm{X}$-polarization (where $\mathrm{X} \in\{\Theta, \Phi\}$ ) maximizes the array gain over all possible beam weights and this array gain in $(\theta, \phi)$ is given as

$$
G_{\mathrm{mrc}, \mathrm{X}}(\theta, \phi)=\max _{\left\{\alpha_{i}\right\}: \sum_{i=1}^{N}\left|\alpha_{i}\right|^{2}=1}\left|\sum_{i=1}^{N} \alpha_{i}^{\star} \mathrm{E}_{\mathrm{X}, i}(\theta, \phi)\right|^{2} .
$$

It is straightforward to check that the solution to the problem in (2) is polarization and $(\theta, \phi)$-specific and is given as

$$
\alpha_{i}=\frac{\mathrm{E}_{\mathbf{X}, i}(\theta, \phi)}{\left\|\mathbf{E}_{\mathbf{X}}(\theta, \phi)\right\|}=\frac{\mathrm{E}_{\mathbf{X}, i}(\theta, \phi)}{\sqrt{\sum_{i=1}^{N}\left|\mathrm{E}_{\mathbf{X}, i}(\theta, \phi)\right|^{2}}}
$$

where $\|\cdot\|$ denotes the two-norm of a vector. We thus have

$$
G_{\mathrm{mrc}, \mathrm{x}}(\theta, \phi)=\sum_{i=1}^{N}\left|\mathrm{E}_{\mathrm{X}, i}(\theta, \phi)\right|^{2} .
$$

- Scheme 2: The second scheme considered corresponds to EGC which is similar to the MRC scheme except that the beamformer has an equal gain amplitude constraint

\footnotetext{
${ }^{5}$ Note that every direction in the sphere can be uniquely specified by a $(\theta, \phi)$ angle pair. The unit-norm vector from the center of the sphere to this point is specified as $[\sin (\theta) \cos (\phi) \sin (\theta) \sin (\phi) \cos (\theta)]$. This classical coordinate system/transformation is also used in 3GPP channel/antenna modeling studies [5].
}

for all the antennas. It is straightforward to note that the solution to this problem is given as

$$
G_{\mathrm{egc}, \mathrm{x}}(\theta, \phi)=\frac{1}{N}\left(\sum_{i=1}^{N}\left|\mathrm{E}_{\mathrm{X}, i}(\theta, \phi)\right|\right)^{2} .
$$

In terms of the performance comparison between MRC and EGC, we have

$$
\begin{aligned}
& \sum_{i=1}^{N}\left|\mathrm{E}_{\mathrm{X}, i}(\theta, \phi)\right|^{2}=G_{\mathrm{mrc}, \mathrm{X}}(\theta, \phi) \\
& \quad \geq G_{\mathrm{egc}, \mathrm{X}}(\theta, \phi)=\frac{1}{N}\left(\sum_{i=1}^{N}\left|\mathrm{E}_{\mathrm{X}, i}(\theta, \phi)\right|\right)^{2} .
\end{aligned}
$$

This conclusion is easy to establish and it is as intuitively expected since the space of optimization (amplitude and phase optimization) for MRC is bigger than that for EGC (phase-only optimization).

- Scheme 3: In contrast to the above two schemes, the third scheme is designed keeping in mind a practical implementation. This scheme corresponds to the use of a finite-sized RF/analog beam codebook for beamforming. Note that in the 5G-NR beam acquisition process [27], directional beams providing sectoral coverage are scanned at the base-station end, while the UE uses one fixed beam from the codebook as the base-station runs through its beams. This process is repeated till the UE can find the best beam pair for itself (from the codebook) and the base-station, and convey this information back to the base-station. Thus, the worst-case beam acquisition overhead with a codebook-based scheme is proportional to the size of the UE codebook. To minimize the initial acquisition overhead, the UE uses a pseudo-omni beam for each sector with a subarray of choice for this sector ( $\mathrm{P}-1$ phase). Beam refinement follows by local search and optimization around the beam pair link established in the $\mathrm{P}-1$ phase (the base-station refinement is called the P-2 phase and the UE refinement is called the P-3 phase in [27]).

While more beams (at both the base-station and UE ends) can result in better array gains and hence better link performance, it comes at the cost of a higher beam acquisition overhead. Therefore, for a specific UE design, a codebook size is optimally picked to tradeoff beam acquisition overhead with link performance (see Sec. III-B for details). The use of the best beam from the codebook is expected to result in good spherical coverage performance. The array gain performance over a direction $(\theta, \phi)$ with a size- $K$ codebook of beams over an $N$ antenna element subarray $\left(w_{i j}, i=\right.$ 
$1, \cdots, N, j=1, \cdots, K)$ is given as

$$
\begin{aligned}
G_{\mathrm{cbk}, \mathrm{x}}(\theta, \phi)= & \max w_{i j, j=1, \cdots, K}\left|\sum_{i=1}^{N} w_{i j}^{\star} \mathrm{E}_{\mathrm{X}, i}(\theta, \phi)\right| \\
& : \sum_{i=1}^{N}\left|w_{i j}\right|^{2}=1
\end{aligned}
$$

Note that as $K \rightarrow \infty$, the codebook performance can approach that of the MRC scheme. That is, $G_{\mathrm{cbk}, \mathrm{x}}(\theta, \phi) \rightarrow G_{\mathrm{mrc}, \mathrm{x}}(\theta, \phi)$ for every $(\theta, \phi)$ and both polarizations. Similarly, if the codebook entries are constrained to have equal amplitude and further if $K \rightarrow \infty$, we have $G_{\mathrm{cbk}, \mathrm{x}}(\theta, \phi) \rightarrow G_{\mathrm{egc}, \mathrm{x}}(\theta, \phi)$ for every $(\theta, \phi)$ and both polarizations.

- Scheme 4: The fourth scheme corresponds to selecting the best single antenna element (from amongst all the possible antennas across all the antenna modules at the $\mathrm{UE}$ side) for a direction $(\theta, \phi)$ to result in:

$$
G_{\text {ant sel, } \mathrm{X}}(\theta, \phi)=\max _{i=1, \cdots, N}\left|\mathrm{E}_{\mathbf{X}, i}(\theta, \phi)\right|^{2} .
$$

Since no beamforming array gain is realized with this scheme and the gains are purely from antenna selection, this scheme is pessimistic in terms of the available antenna capabilities and corresponds to a legacy beamforming solution such as those available in prior generations (e.g., $3 \mathrm{G}$ or $4 \mathrm{G}$ ) of most wireless devices.

The four schemes introduced above are studied in terms of their selection diversity performance in this work. For this, we use the following metric:

$G_{\text {scheme, total }}(\theta, \phi)=G_{\text {scheme, } \Theta}(\theta, \phi)+G_{\text {scheme }, \Phi}(\theta, \phi)$

where $G_{\text {scheme, } \mathrm{X}}(\theta, \phi)$ denotes the array gain in the $\mathrm{X}$ polarization with $\mathrm{X} \in\{\Theta, \Phi\}$. In (9), $G_{\text {scheme, total }}(\theta, \phi)$ captures the total array gain seen in both polarizations in the direction $(\theta, \phi)$. This metric is used instead of choices such as

$G_{\text {scheme } \max }(\theta, \phi)=\max \left(G_{\text {scheme }, \Theta}(\theta, \phi), G_{\text {scheme }, \Phi}(\theta, \phi)\right)$.

While the metrics in (9) and (10) are equivalent (or comparable) in the boresight direction and its vicinity for a certain subarray, 10 can severely underestimate performance over the edge of the coverage area of the subarray since signal strengths over both polarizations may be comparable at these points. Over these points, gains over both polarizations may be combined with a polarization combining scheme (such as Alamouti coding, cyclic delay diversity, etc.) and the metric in 9 appears to reflect the true selection diversity capabilities.

\section{B. RF/Analog Beam Codebook Design}

As noted in Sec. III-A with hierarchical beamforming as in $5 \mathrm{G}-\mathrm{NR}$, the $\mathrm{P}-1$ phase is typically 6 performed over a burst set of sychronization signal blocks (SSBs) [45]. Beamformed transmissions over different beams (up to 64 beams are allowed in 5G-NR for millimeter wave frequencies) can be used over multiple SSBs in a burst set of $5 \mathrm{~ms}$ duration and the burst set can be repeated with periodicity that is one of either $5 \mathrm{~ms}, 10 \mathrm{~ms}$, or $20 \mathrm{~ms}$. With initial practical implementations as well as with the initial acquisition phase of UEs, it is expected that the SSB burst set periodicity is set to $20 \mathrm{~ms}$ (which is assumed in this work). On the other hand, P-2 and P-3 beam refinements are typically performed with aperiodic CSI-RS symbols in a UE-specific manner. In some possibilities with low-cost and low-complexity basestations, P-3 beam refinement can also be performed over SSB signals.

We assume that both the base-station and all the UE designs considered in this work are powered by two RF chains, where each RF chain is excited by one (orthogonal) polarization. This is a reasonable assumption for the initial generation of millimeter wave systems. That is, independent beam weights can be set for either of the two RF chains corresponding to different subarrays excited by these RF chains. Thus, two subarrays at the UE side can be beam trained by the base-station at the same time. With UE side beam switching constraints in mind, we assume that the UE uses a single pseudo-omni beam per subarray over each SSB burst set. Further, with UE side power-performance tradeoffs in mind, a single antenna exciting pseudo-omni beam is used from all the subarrays for the P-1 phase. While more complicated pseudo-omni beam choices can be used, the considered design is representative of practical implementations. Thus, for the face design with 8 subarrays over two polarizations, the initial beam acquisition overhead in the $\mathrm{P}-1$ phase is $20 \mathrm{~ms} \times 8$ subarrays $/ 2$ polarizations, which equals $80 \mathrm{~ms}$. Similarly, for the edge design with 6 subarrays, the initial beam acquisition overhead corresponds to $60 \mathrm{~ms}$. These numbers appear to be representative of the initial beam acquisition overheads expected in interoperability development and testing trials, and commercial operations of $5 \mathrm{G}-\mathrm{NR}$.

In terms of peak performance, in order to compare the UE designs introduced in Sec. II-A it is important to perform a fair comparison of the codebook-based beamforming scheme across these designs. With different codebook sizes, the beam acquisition latencies can be different. To address these concerns, for the face design, 4 beams are used for each polarization of the $2 \times 2$ dual-polarized patch subarrays, and 2 beams are used for each dipole subarray leading to 12 beams per antenna module as well as 12 beams per

\footnotetext{
${ }^{6}$ Note that the initial beam acquisition can also be performed over periodically configured channel state information reference signals (CSI$\mathrm{RS})$.
} 




(a)



(b)

Fig. 2. View of codebooks' array gain performance over the sphere (represented in the $\theta-\phi$ Cartesian plane) for the (a) face and (b) edge designs.

polarization. For the edge design, 4 beams are used for each polarization of the $4 \times 1$ dual-polarized patch subarray for 8 beams per module and 12 beams per polarization. Since the codebook sizes are 24 for both the designs, the performance of these two UE designs can be compared fairly. Note that while more complicated and different-sized codebooks can be considered for the two designs and their performance can be compared with some performance penalty function (e.g., a $3 \mathrm{~dB}$ penalty for a doubling of the codebook in one design relative to the other, etc.), the method proposed here is reasonable for practical implementations.

If UE-specific CSI-RS symbols are used for beam refinement, since multiple symbols can be configured for CSI-RS in a downlink-specific subframe, P-3 beam refinement can be performed within 1-2 slots even under the assumption of multi-symbol averaging for signal-to-noise ratio (SNR) enhancement. As an illustration, since each subarray has at most 4 refined/narrow beams for a pseudo-omni beam, assuming a four symbol averaging, we would need at most 16 symbols which can be accommodated within 2 slots since 14 symbols make a slot. Under a $60 \mathrm{kHz}$ subcarrier spacing for millimeter wave transmissions, the worst-case beam refinement overhead is thus less than $0.50 \mathrm{~ms}$ (which is significantly smaller than that accrued in the P-1 phase). Alternately, if SSBs at $20 \mathrm{~ms}$ periodicity are used for P-3 beam refinement, the worst-case beam refinement overhead for the P-3 phase (assuming 4 narrow beams) is $20 \mathrm{~ms}$ $\times 4$ beams/1 polarization, which equals an additional $80 \mathrm{~ms}$ time-period. Note that in this calculation, beam refinement is constrained to the RF chain corresponding to the selected subarray (unlike the initial beam acquisition phase over two RF chains).

The individual beam weights in the codebook can be optimally designed to cover certain angular regions over the sphere. While the beam design process in itself can be implementation-specific or proprietary, general design principles are expounded in [34]. In this work, the beams for the $4 \times 1$ subarrays are designed such that each beam results in a beamwidth of $\approx 30^{\circ}$. Similarly, the beams for the $2 \times 2$ and $2 \times 1$ subarrays have a beamwidth of $\approx 55^{\circ}$. All the beam weights (for either design) are constrained to meet an equal amplitude and a five-bit phase shifter resolution. Fig. 2 presents the array gain performance with the codebooks over the sphere (represented on a $\theta-\phi$ Cartesian plane) for the face and edge designs. For each point $(\theta, \phi)$ over the sphere, the best representative from each design's codebook is used here. Clearly, from Fig. 2, we observe that the codebooks are designed to meet good array gain performance over a significant fraction of the sphere.

\section{Blockage Modeling}

Hand and body blockage can be substantial at millimeter wave carrier frequencies (relative to sub- $6 \mathrm{GHz}$ frequencies) since the size of many physically small objects in the proximity of the antennas become electrically comparable with the wavelength of propagation.

In the 3GPP channel modeling document TR 38.901, a blockage model is proposed [5, pp. 53-57] to capture these detrimental effects under two variants: a stochastic variant (Option A) and a map-based/ray tracing-based variant (Option B). The stochastic variant proposes a spherical coverage blockage tailored to the hand in Portrait or Landscape orientations around a UE modeled to form factor considerations. As illustrated in Table III this model (labeled "Model 1" in this work) is captured by the center of the blocker $\left(\phi_{1}\right.$, $\left.\theta_{1}\right)$, and the angular spread of the blocker $\left(x_{1}, y_{1}\right)$ in azimuth and elevation with the blocking angles captured as $\phi \in\left[\phi_{1}-\frac{x_{1}}{2}, \phi_{1}+\frac{x_{1}}{2}\right]$ and $\theta \in\left[\theta_{1}-\frac{y_{1}}{2}, \theta_{1}+\frac{y_{1}}{2}\right]$ in azimuth and elevation, respectively. Over this spatial region, a simplistic flat $30 \mathrm{~dB}$ loss is assumed.

It is understood that the 3GPP blockage model is quite pessimistic [29] relative to form factor UE designs due to the use of horn antenna measurements (with smaller beamwidths) to model hand blockage loss. In this context, 
based on $28 \mathrm{GHz}$ experimental prototype studies [28], a modified blockage model (labeled as "Model 2" in this work) is proposed in [29]. In this model, the spatial blockage region is retained from the $3 \mathrm{GPP}$ model and a log-normal blockage loss term, as summarized in Table II is used. We study the spherical coverage CDFs with these two blockage models in this work.

At this stage, it is to be noted that the dielectric properties of the skin tissue (such as the relative dielectric constant and conductivity) determine the penetration depth of the electromagnetic radiation into the hand and its reflection. At $28 \mathrm{GHz}$, it is observed that the penetration depth into the hand is very small and a significant fraction of the energy is reflected. The hand blockage loss is a function of the shape of the hand (its curvature, roughness of skin tissue, etc.) and the model presented in [29] and used in this work is reflective of these ensemble trends. That said, the precise reflection response and penetration loss of different materials to millimeter wave frequencies is a function of the material, incidence angle and polarization [10, Sec. IV-A and Fig. 5]. While similar overall behaviors are seen with the hand to the two polarizations, more work is necessary to understand the precise differences, if any. Thus, the hand blockage model used here can be seen to be a good first effort at understanding the impact of hand impairments for UE design.

\section{Spherical Coverage CDF TradeoffS}

We now present results on spherical coverage CDF tradeoffs with the two UE designs considered in this work. For this, as explained in Sec. III individual antenna element response functions in both polarizations are computed using the HFSS commercial software suite [26] with a $1^{\circ}$ precision in azimuth and elevation. Beamforming gains are computed with MRC, EGC, an RF/analog beam codebook and single antenna selection with the diversity performance metric as described in Sec. III-A. Spherical coverage CDF is computed as described in Appendix $\mathrm{A}$

\section{A. Freespace Performance}

In our first study, in Figs. 3 (a)-(b), we describe the beamforming array gain tradeoffs for the UE designs with these four schemes in Freespace (that is, with no hand blockage). With both the designs, we observe that the EGC scheme performs as well as the MRC scheme over the entire sphere. This conclusion implies that phase-only control is sufficient to obtain the optimal spherical coverage and the cost associated with amplitude control can be forsaken with minimal performance penalties. This also motivates the design of RF/analog beam codebooks with only phase shifter control as done in Sec. III-B. This conclusion stems from the fact that all the antennas that make a certain subarray have similar/comparable amplitudes over the whole sphere and no specific antenna sees an anomalous behavior (relative to others) necessitating amplitude control.

For both the UE designs, the RF/analog beam codebooks are within 1-2 $\mathrm{dB}$ of the MRC/EGC performance suggesting the goodness of the codebook design principles. However, the worst-case points of the codebook's performance are $7 \mathrm{~dB}$ and $10 \mathrm{~dB}$ away from the peak gain for the face and edge designs, respectively. While this observation could suggest that there are significant gaps relative to MRC/EGC performance, this is a naïve conclusion that needs to be tested with real impairments. We will see subsequently that both the face and edge designs are competitive with hand blockage.

With the edge design, single antenna selection is approximately 5-6 dB worse than MRC/EGC. This gap can be explained as the co-phasing gain from four antenna subarrays used in this design. On the other hand, with the face design, this gap reduces from $6 \mathrm{~dB}$ at the peak to $3 \mathrm{~dB}$ at the tail corresponding to the switch from a $2 \times 2$ patch subarray to a $2 \times 1$ dipole subarray. In terms of codebook performance relative to $\mathrm{MRC} / \mathrm{EGC}$, the edge design shows a near-constant gap over the CDF curve $(\approx 1 \mathrm{~dB})$. On the other hand, the face design appears to have a gap that increases from the peak to the tail. This can be attributed to: i) loss in array gain as we move from the beams' boresight steering direction to the edge of coverage of each beam, and ii) switch from a four element subarray to a two element subarray. From a pictorial view of the codebooks in Fig. 2, we observe that the edge design has coverage holes only/mostly over the poles (which are discounted with the $\sin (\theta)$ factor in the spherical coverage computation - See Appendix A), whereas the face design has coverage holes at random points over the sphere accounting for the degradation in codebook performance from the peak to the tail.

\section{B. Performance of the Face Design with Hand Blockage}

We now study the performance of the face design with the blockage models described in Sec. III-C. Figs. 4 (a)-(b) present the performance of the different schemes with the two blockage models in Portrait mode, whereas Figs. 4 (c)-(d) present the performance with the two models in Landscape mode.

From Table III the blockage region in Portrait and Landscape modes occupy the following fraction of physical/spatial angles:

$$
\begin{gathered}
\text { Physical angle loss }\left.\right|_{\text {Portrait }}=\frac{120^{\circ} \times 80^{\circ}}{360^{\circ} \times 180^{\circ}}=14.81 \% \\
\text { Physical angle loss }\left.\right|_{\text {Landscape }}=\frac{160^{\circ} \times 75^{\circ}}{360^{\circ} \times 180^{\circ}}=18.52 \%,
\end{gathered}
$$

respectively. Since the spatial angles need to be weighted based on the Jacobian (see Appendix (A), these blocked 
TABLE II

Hand Blockage Models CONSIDEREd In THIS WORK

\begin{tabular}{|c|c|c|c|c|c|}
\hline Scenario & $\phi_{1}$ & $x_{1}$ & $\theta_{1}$ & $y_{1}$ & Blockage loss (in dB) \\
\hline Portrait mode & $\overline{260^{\circ}}$ & $120^{\circ}$ & $100^{\circ}$ & $\overline{80^{\circ}}$ & Model 1 \\
\hline Landscape mode & $40^{\circ}$ & $160^{\circ}$ & $110^{\circ}$ & $75^{\circ}$ & $\mathcal{N}(\mu=15.3 \mathrm{~dB}, \sigma=3.8 \mathrm{~dB})$ \\
\hline
\end{tabular}



(a)



(b)

Fig. 3. Array gain performance of different beamforming schemes in Freespace for the (a) face and (b) edge designs.

angles correspond to a CDF loss of

$$
\begin{aligned}
& \text { CDF loss }\left.\right|_{\text {Portrait }} \\
& =\frac{1}{4 \pi} \int_{\phi=\phi_{\mathrm{p}, 1}}^{\phi_{\mathrm{p}, \mathrm{u}}} \int_{\theta=\theta_{\mathrm{p}, \mathrm{l}}}^{\theta_{\mathrm{p}, \mathrm{u}}} \sin (\theta) \cdot d \theta d \phi=21.07 \% \\
& \text { CDF loss }\left.\right|_{\text {Landscape }} \\
& =\frac{1}{4 \pi} \int_{\phi=\phi_{\mathrm{l}, \mathrm{l}}}^{\phi_{\mathrm{l}, \mathrm{u}}} \int_{\theta=\theta_{\mathrm{l}, \mathrm{l}}}^{\theta_{\mathrm{l}, \mathrm{u}}} \sin (\theta) \cdot d \theta d \phi=26.00 \% \text {, }
\end{aligned}
$$

where $\phi_{\mathrm{p}, \mathrm{I}}=200^{\circ} \cdot \pi / 180, \phi_{\mathrm{p}, \mathrm{u}}=320^{\circ} \cdot \pi / 180, \theta_{\mathrm{p}, \mathrm{l}}=$ $60^{\circ} \cdot \pi / 180, \theta_{\mathrm{p}, \mathrm{u}}=140^{\circ} \cdot \pi / 180$, and $\phi_{\mathrm{l}, \mathrm{I}}=-40^{\circ} \cdot \pi / 180$, $\phi_{\mathrm{l}, \mathrm{u}}=120^{\circ} \cdot \pi / 180, \theta_{\mathrm{l}, \mathrm{l}}=72.5^{\circ} \cdot \pi / 180, \theta_{\mathrm{l}, \mathrm{u}}=147.5^{\circ}$. $\pi / 180$. The performance degradation in the tails of the Portrait and Landscape modes correspond to the CDF loss region estimates in (13) and (14), as expected. The major difference between the two sets of curves is that the flat 30 $\mathrm{dB}$ loss assumed with the 3GPP model (Model 1) renders the tail region completely irretrievable and the performance loss over this region is abrupt/dramatic. On the other hand, with a log-normal loss model (Model 2), this loss in performance is smoother allowing for some recovery over certain directions. Nevertheless, in general, it appears that blockage leads to a bimodal behavior of almost no loss over the unblocked region and essentially irretrievable loss over the blocked region.

\section{Freespace vs. Blockage}

Figs. 5 and 6 present the comparison between Freespace performance for the two designs and hand blockage in Portrait and Landscape modes with Models 1 and 2, respectively. All the four beamforming schemes are considered in these plots. Fig. 5 reinforces the earlier finding of the blocked region being completely irretrievable, independent of which UE design is used. On the other hand, Fig. 6 shows a smoother degradation over the blocked angles with both the designs and with the precise set/quantum of angles recoverable with blockage depending on the UE design.

\section{Comparisons Across Designs}

Since the face and edge designs are directly and fairly comparable with each other (due to the same codebook sizes), Fig. 7 presents a head-to-head comparison of these designs in Freespace and in Portrait/Landscape modes with blockage. Blockage Model 2 and the RF/analog beam codebook scheme are used in all the studies in Fig. 7 From Fig. 7(a), we observe that while both the designs are comparable in the top 20 percentile points of the sphere in Portrait mode, the edge design appears to be better (by up to $1.5 \mathrm{~dB}$ ) over the next 35 percentile points. The face design appears to be better over the remaining $\approx 20$ percentile points before blockage effects kick in.

While both the face and edge designs are blocked over approximately $21 \%$ of the sphere in the Portrait mode (see (13), their crossovers can be explained by the following 




(a)

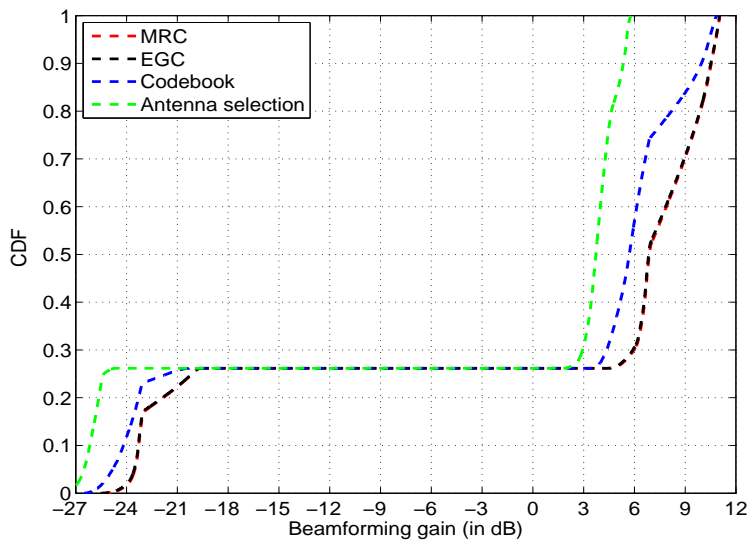

(c)



(b)



(d)

Fig. 4. Array gain performance of the face design with blockage model in (a)-(b) Portrait mode (Models 1 and 2) and (c)-(d) Landscape mode (Models 1 and 2).



(a)



(b)

Fig. 5. Array gain performance with Freespace and blockage in Portrait and Landscape (Model 1) for the (a) face and (b) edge designs.

observations: Approximate beamforming array gain with the $\mathrm{RF}$ codebook at the $70^{\text {th }}$ percentile point for the face and edge designs are 7 and $8.5 \mathrm{~dB}$, respectively. Similar numbers for the $50^{\text {th }}$ and $30^{\text {th }}$ percentile points are $6.5 \mathrm{vs} .7 \mathrm{~dB}$ and 


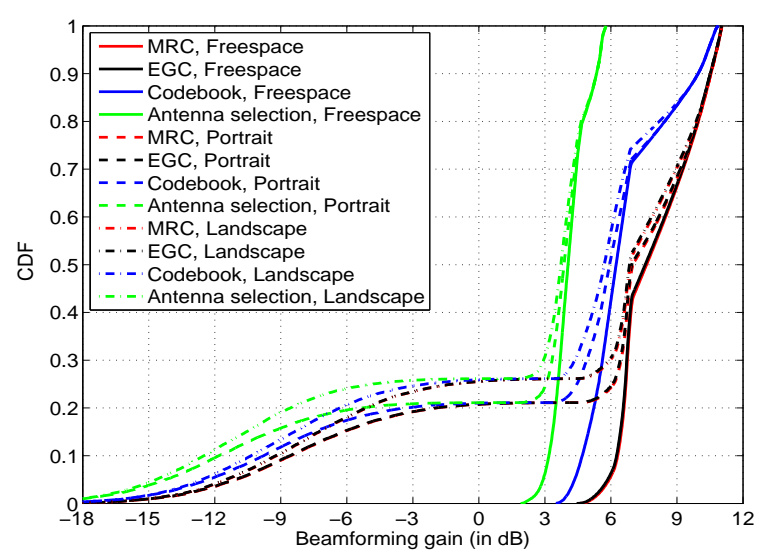

(a)



(b)

Fig. 6. Array gain performance with Freespace and blockage in Portrait and Landscape (Model 2) for the (a) face and (b) edge designs.

5.5 vs. $5 \mathrm{~dB}$, respectively. This tradeoff arises due to the structure of antenna arrays $(2 \times 2$ planar arrays and $2 \times 1$ linear arrays in the face design vs. $4 \times 1$ linear arrays in the edge design). The better relative performance of the edge design over the face design in the middle 35 percentile points and its reversal in the next 20 percentile points is directly a result of the array gain tradeoffs.

On the other hand, the mismatch between the area blocked with the hand in the right-hand Landscape mode (top short edge that is totally blocked in the edge design vs. the top front module that is only partially blocked in the face design) means that the face design appears to be uniformly better than the edge design (by up to $1.5 \mathrm{~dB}$ ). From these observations, there does not appear to be an overwhelming advantage (defined as greater than 2-3 dB) for either design suggesting that both designs are comparable in terms of performance and the choice between them should be decided based on implementation tradeoffs as described in Sec. II-B

Finally, system level simulation studies for an indoor channel environment with macroscopic fading parameters as described in [10] are performed. These parameters describe the third and fourth floors of the Qualcomm building in Bridgewater, NJ. Table $\amalg$ illustrates the various parameters used in this comparative study. In particular, a base-station of size $16 \times 4$ using a size $16 \mathrm{RF} /$ analog beamforming codebook (of DFT beams) covering a $120^{\circ} \times 30^{\circ}$ coverage area and UEs according to the face and edge designs with RF/analog beam codebooks as described in Sec. III-B are used in these studies. Note that these codebooks can be considered to represent the performance at the end of the $\mathrm{P}-1 / 2 / 3$ procedures. The spectral efficiencies (per layer) in $\mathrm{bps} / \mathrm{Hz}$ with dual layer polarization-MIMO transmission/reception at $28 \mathrm{GHz}$ are illustrated in Fig. 7 (b). From this study, we observe that the edge and face designs tradeoff performance with each other in Freespace with the edge design being better in the (approximately) top 50 percentile points (by up to $0.3 \mathrm{bps} / \mathrm{Hz}$ or around $1 \mathrm{~dB}$ ) and the face design being better in the (approximately) bottom 50 percentile points. The trends with blockage are as before, reinforcing the conclusions made previously.

TABLE III

System LeVel Simulation Parameters

\begin{tabular}{|c||c|}
\hline Metric & Value used \\
\hline \hline Base-station antenna dimensions & $16 \times 4$ \\
\hline Base-station coverage area & $120^{\circ} \times 30^{\circ}$ \\
\hline Base-station codebook size & 16 \\
\hline UE coverage area & $360^{\circ} \times 180^{\circ}$ \\
\hline UE codebook size & 24 \\
\hline EIRP & $15 \mathrm{dBm}$ \\
\hline Bandwidth & $100 \mathrm{MHz}$ \\
\hline UE noise figure & Indoor, \\
\hline Channel environment & PLE $=3.46, \sigma_{\mathrm{SF}}=8.31 \mathrm{~dB}$ \\
\hline Distance from base-station to UE & $30 \mathrm{~m}$ \\
\hline No. of clusters & 4 \\
\hline \hline
\end{tabular}

\section{E. Generalizations to Other UE Designs}

To study the utility of the above conclusions to other UE designs, we consider two other popular designs in the literature, as illustrated in Fig. 8(a). These designs are

- Design 3: A maximalist edge design with four antenna modules (on four sides of the UE) with each module made of $4 \times 1$ dual-polarized patch subarrays and a $4 \times 1$ dipole subarray. While full spherical coverage can be obtained with patch elements alone, the use of dipole elements provides complementary coverage and hence, better robustness at the expense of cost associated with more antenna elements as well as the control circuitry for these elements.

- Design 4: An L-shaped edge design with four antenna modules (on four sides of the UE) with each module 




(a)



(b)

Fig. 7. Comparative performance between the face and edge designs in Freespace and with blockage (Model 2): (a) Array gain and (b) Spectral efficiency.



(a)



(c)



(b)



(d)

Fig. 8. (a) Pictorial illustration of Designs 3 and 4. Array gain performance with Freespace and blockage in Portrait and Landscape (Model 2) for (b) Design 3 and (c) Design 4. (d) Comparative performance between Designs 3 and 4 with blockage Model 2.

being L-shaped and spanning two adjacent sides of coverage. Each side of coverage is made of $4 \times 1$ dual- 
polarized patch subarrays alone.

Note that a version of Design 3 has been proposed in [22], [24] with dipole antennas instead of dual-polarized patches and dipoles, which is a minor design enhancement. A number of features of Design 4 can be seen in other designs such as [14], [17], [20], [23], [24], [39], as well as [40].

Since both these designs have four antenna modules and have far more subarrays (12 and 16, respectively) than either the face or edge designs ( 8 and 6 , respectively), we use an RF codebook of larger size (size of 48) than those used with the face and edge designs (size of 24). Note that a smaller codebook size with Designs 3 and 4 can lead to coverage holes with poor spherical coverage tradeoffs. For Design 3, we use 4 beams for each polarization of the patch and dipole subarray corresponding to 12 beams per antenna module for a codebook size of 48 . For Design 4 , we use 3 beams for each polarization of the patch corresponding to 12 beams per antenna module, also for a codebook size of 48 . For these two designs, Figs. 8 b)(c) present the beamforming array gain comparison with the four beamforming schemes in Freespace and with hand blockage in Portrait and Landscape modes using Model 2. Similar to the face and edge designs, we observe that for both Designs 3 and 4, the RF/analog beam codebooks are within 1-2 $\mathrm{dB}$ of the MRC/EGC performance suggesting the goodness of the codebook design principles. In particular, the worst-case points of the codebook's performance in Freespace are $3 \mathrm{~dB}$ and $6 \mathrm{~dB}$ away (which is better than the face and edge designs) from the peak gain for these designs. As intuitively expected from a co-phasing with four antennas in either design, single antenna selection is approximately 5 $6 \mathrm{~dB}$ worse than MRC/EGC. Blockage tradeoffs for both the designs are similar to those described earlier for the face and edge designs.

Since Designs 3 and 4 are directly and fairly comparable with each other, Fig. 8d d) provides a comparison across these two designs. From this study, we observe that Design 3 has a universally (albeit slightly) better performance (in Freespace as well as with blockage) over Design 4. This plot suggests that the use of dipoles over patches that scan the other side of the $\mathrm{L}$ can result in a better performance for diversity. Thus, the use of the appropriate/correct antenna modules is crucial for good performance in millimeter wave systems.

\section{CONCLUding REMARKS}

The focus of this paper has been on the study of spherical coverage CDF of two popular millimeter wave UE designs with real impairments such as hand blockage. The designs considered in this paper correspond to a face design and an edge design, respectively. For our studies, we considered four types of beamforming schemes (MRC, EGC, RF/analog beam codebook and antenna selection) with two types of blockage models (3GPP blockage model and a modified version of the 3GPP model). From our studies, we established the overhead of beam training as being the key determinant (and not the "theoretical" capabilities enabled with multiple antenna modules) for robust spherical coverage performance. That is, it is not merely sufficient that the UE is packed with a large number of antenna modules, but that the subarrays in these modules have to be scanned/learned with an appropriately designed beam codebook in a practical implementation. Further, the size of a good codebook has to scale with the number of antenna modules and can render the coverage gains unrealizable from a practical standpoint. From this view, we established the goodness of the edge UE design that also has other additional advantages such as low cost and power consumption, implementation ease, and minimal exposure related challenges [33]. Table IV provides a broad overview and summary of the major issues with these designs.

That said, this work has barely scratched the surface in terms of coupling different practical/commercial UE design challenges with their system level impacts. In fact, this work has exposed the challenges of good UE designs for millimeter wave transmissions, which are quite unlike those of sub-6 GHz systems. More work is necessary to understand the impact of optimal codebook construction/beamforming schemes on spherical coverage, incorporating priors (e.g., base-station downtilt, UE modalities, etc.) on spatial angles in spherical coverage studies, initial acquisition vs. steadystate performance tradeoffs, etc. Further work is also necessary in incorporating metrics that capture multiple layer/RF chain performance and establishing the structure of optimal UE designs for such metrics. Leveraging antenna response functions in a UE design incorporated with practical display and frame materials such as glass, plastic, ceramic, etc., are also important in future studies.

\section{APPENDIX}

\section{A. Computing Spherical Coverage CDF}

Let $G_{\text {scheme, total }}(x, y, z)$ denote the total array gain (over both polarizations) of a certain beamforming scheme at a point $(x, y, z)$ represented in the $\mathrm{X}-\mathrm{Y}-\mathrm{Z}$ Cartesian coordinate system. Then, the CDF of spherical coverage evaluated at $\alpha$ over a sphere of radius $R$ is given as

$$
F(\alpha)=\frac{\iiint \mathbb{1}\left(G_{\text {scheme, total }}(x, y, z) \leq \alpha\right) d x d y d z}{\iiint d x d y d z}
$$

where $\mathbb{1}(\bullet)$ denotes the indicator function of the underlying variable. The differential element in the Cartesian coordinate system is transformed to the differential element in the spherical coordinate system (with $x=r \sin (\theta) \cos (\phi), y=$ $r \sin (\theta) \sin (\phi)$ and $z=r \cos (\theta))$ as

$$
d x d y d z=\mathcal{J} d r d \theta d \phi
$$

where $\mathcal{J}=|\operatorname{det}(J)|$ with $J$ denoting the Jacobian matrix of the transformation, as described in (17) at the top of the 


$$
\begin{aligned}
J & =\left[\begin{array}{lll}
\frac{\partial x}{\partial r} & \frac{\partial x}{\partial \theta} & \frac{\partial x}{\partial \phi} \\
\frac{\partial y}{\partial r} & \frac{\partial y}{\partial \theta} & \frac{\partial y}{\partial \phi} \\
\frac{\partial z}{\partial r} & \frac{\partial z}{\partial \theta} & \frac{\partial z}{\partial \phi}
\end{array}\right]=\left[\begin{array}{ccc}
\sin (\theta) \cos (\phi) & r \cos (\theta) \cos (\phi) & -r \sin (\theta) \sin (\phi) \\
\sin (\theta) \sin (\phi) & r \cos (\theta) \sin (\phi) & r \sin (\theta) \cos (\phi) \\
\cos (\theta) & -r \sin (\theta) & 0
\end{array}\right] \\
F(\alpha) & =\frac{\int_{r=0}^{R} \int_{\theta=0}^{\pi} \int_{\phi=0}^{2 \pi} \mathbb{1}\left(G_{\text {scheme, total }}(\theta, \phi) \leq \alpha\right) r^{2}|\sin (\theta)| d r d \theta d \phi}{\int_{r=0}^{R} \int_{\theta=0}^{\pi} \int_{\phi=0}^{2 \pi} r^{2}|\sin (\theta)| d r d \theta d \phi} \\
& =\frac{\int_{\theta=0}^{\pi} \int_{\phi=0}^{2 \pi} \mathbb{1}\left(G_{\text {scheme, total }}(\theta, \phi) \leq \alpha\right) \sin (\theta) d \theta d \phi}{4 \pi} .
\end{aligned}
$$

\begin{tabular}{|c|c|c|}
\hline Issue of interest & Face design & Edge design \\
\hline \multicolumn{3}{|c|}{ Design/cost/regulatory tradeoffs } \\
\hline Mounting problems & High & Low \\
\hline No. of antenna modules & 2 & 3 \\
\hline Exposure-related challenges & Major & Minor \\
\hline Dipole-related pros/cons & Yes & No \\
\hline \multicolumn{3}{|c|}{ Beam scanning complexity } \\
\hline Worst-case initial acquisition overhead & $80 \mathrm{~ms}$ & $60 \mathrm{~ms}$ \\
\hline Beam localization with 2D arrays & Possible with patches & Not possible \\
\hline \multicolumn{3}{|c|}{ Beamforming performance } \\
\hline Link budget needed to overcome penetration & More & Less \\
\hline Freespace performance at cell center & Poor & Better \\
\hline Freespace performance at cell edge & Better & Poor \\
\hline Performance with Portrait blockage & Better from 55 th to 75 th percentiles & Better from 20 th to 55 th percentiles \\
\hline Performance with Landscape blockage & Universally better & Universally poorer \\
\hline
\end{tabular}

TABLE IV

BROAD TRADEOFFS For THE DifFERENT UE DESIGNS

previous page, resulting in $\mathcal{J}=r^{2}|\sin (\theta)|$. With this, (15) transforms to the description in (18)-(19) at the top of the previous page. It is critical to note the scaling factor $\sin (\theta)$ in 193 which reduces the weightage of points at the poles (where $\theta=0$ and $\pi$ ) and increases the weightage of points at the equator (where $\theta=\pi / 2$ ).

\section{REFERENCES}

[1] F. Khan and Z. Pi, "An introduction to millimeter wave mobile broadband systems," IEEE Commun. Magaz., vol. 49, no. 6, pp. 101107, June 2011.

[2] N. Bhushan, J. Li, D. Malladi, R. Gilmore, D. Brenner, A. Damnjanovic, R. T. Sukhasvi, C. Patel, and S. Geirhofer, "Network densification: The dominant theme for wireless evolution into $5 \mathrm{G}$," IEEE Commun. Magaz., vol. 52, no. 2, pp. 82-89, Feb. 2014.

[3] T. S. Rappaport, S. Sun, R. Mayzus, H. Zhao, Y. Azar, K. Wang, G. N. Wong, J. K. Schulz, M. K. Samimi, and F. Gutierrez, "Millimeter wave mobile communications for $5 \mathrm{G}$ cellular: It will work!," IEEE Access, vol. 1, pp. 335-349, 2013.

[4] F. Boccardi, R. W. Heath, Jr., A. Lozano, T. L. Marzetta, and P. Popovski, "Five disruptive technology directions for 5G," IEEE Commun. Magaz., vol. 52, no. 2, pp. 74-80, Feb. 2014.

[5] 3GPP TR 38.901 V14.3.0 (2017-12), "Technical Specification Group Radio Access Network; Study on Channel Model for Frequencies from 0.5 to $100 \mathrm{GHz}$ (Rel. 14)," Dec. 2017.

[6] Aalto University, AT\&T, BUPT, CMCC, Ericsson, Huawei, Intel, KT Corporation, Nokia, NTT DOCOMO, NYU, Qualcomm, Samsung, U. Bristol, and USC, "White paper on "5G channel model for bands up to $100 \mathrm{GHz}$," v2.3, Oct. 2016, Available: [Online]. http://www . 5gworkshops . com/5GCM.html.
[7] A. Maltsev et al., "Channel models for $60 \mathrm{GHz}$ WLAN systems, doc: IEEE 802.11-09/0334r8," May 2010, Available: [Online]. https://mentor.iee.org/802.11/documents.

[8] A. Maltsev et al., "Channel models for IEEE 802.11ay, doc: IEEE 802.11-15/1150r9," May 2016, Available: [Online]. http://www . ieee802.org/11/Reports/tgay_update.htm.

[9] METIS 2020, "METIS channel model, Deliverable D1.4v3," July 2015, Available: [Online]. https://www.metis2020.com/wp-content/uploads/ deliverables/METIS_D1.4_v3.pdf.

[10] V. Raghavan, A. Partyka, L. Akhoondzadeh-Asl, M. A. Tassoudji, O. H. Koymen, and J. Sanelli, "Millimeter wave channel measurements and implications for PHY layer design," IEEE Trans. Ant. Propagat., vol. 65, no. 12, pp. 6521-6533, Dec. 2017.

[11] S. Hur, S. Baek, B. Kim, Y. Chang, A. F. Molisch, T. S. Rappaport, K. Haneda, and J. Park, "Proposal on millimeter-wave channel modeling for 5G cellular system," IEEE Journ. Sel. Topics in Sig. Proc., vol. 10, no. 3, pp. 454-469, Apr. 2016.

[12] M. Shafi, A. F. Molisch, P. J. Smith, T. Haustein, P. Zhu, P. D. Silva, F. Tufvesson, A. Benjebbour, and G. Wunder, "5G: A tutorial overview of standards, trials, challenges, deployment, and practice," IEEE Journ. Sel. Areas in Commun., vol. 35, no. 6, pp. 1201-1221, June 2017.

[13] 3GPP TR 38.803 V14.2.0 (2017-09), “Technical Specification Group Radio Access Network; Study on new radio access technology: Radio Frequency (RF) and co-existence aspects (Release 14)," Sept. 2017.

[14] Apple and Intel, "R4-1713850, Consideration of EIRP spherical coverage requirement," 3GPP TSG-RAN WG4 Meeting \#85, Reno, NV, Nov.-Dec. 2017.

[15] Samsung, Apple, and Intel, "R4-1711036, Consideration of EIRP spherical coverage requirement," 3GPP TSG-RAN WG4 Meeting \#84bis, Dubrovnik, Croatia, Oct. 2017. 
[16] NTT DOCOMO, "R4-1709394, EIRP CDF for mmWave UE" 3GPP TSG-RAN WG4 Meeting NR ad-hoc \#3, Nagoya, Japan, Sept. 2017.

[17] Qualcomm, "R4-1712381, Spherical coverage of realistic design," 3GPP TSG-RAN WG4 Meeting \#85, Reno, NV, Nov.-Dec. 2017.

[18] Qualcomm, "R4-1804587, On UE spherical coverage with glass packaging," 3GPP TSG-RAN WG4 Meeting \#86-bis, Melbourne, Australia, Apr. 2018.

[19] Sony, "R4-1802868, UE spherical coverage at mmWave $28 \mathrm{GHz}$," 3GPP TSG-RAN WG4 \#86, Athens, Greece, Feb.-Mar. 2018.

[20] Sony, "R4-1711424, UE power class and spherical coverage for mmWave $28 \mathrm{GHz}, " 3$ GPP TSG-RAN WG4 Meeting \#84-bis, Dubrovnik, Croatia, Oct. 2017.

[21] Sony, "R4-1807490, UE spherical coverage measurements at mmWave 28 GHz," 3GPP TSG-RAN WG4 Meeting \#87, Busan, South Korea, May 2018

[22] Sony, "R4-1707330, On UE mmWave antennas," 3GPP TSG-RAN WG4 Meeting \#84, Berlin, Germany, Aug. 2017.

[23] Sony and Ericsson, "R4-1800888, UE spherical coverage at mmWave 28 GHz," 3GPP TSG-RAN WG4 Meeting AH-1801, San Diego, CA, Jan. 2018

[24] Sony and Ericsson, "R4-1706562, UE EIRP for mmWave $28 \mathrm{GHz}$," 3GPP TSG-RAN WG4 Meeting \#83, Hangzhou, China, May 2017.

[25] LG Electronics, "R4-1806676, Measurement EIRP levels for spherical coverage of NR UE at FR2," 3GPP TSG-RAN WG4 Meeting \#87, Busan, South Korea, May 2018.

[26] Ansys, "High-Frequency antenna response/Structure Simulator (HFSS)," Available: [Online]. https://www.ansys.com/products/electronics/ansys-hf ss.

[27] 3GPP TR 38.912 V14.1.0 (2017-06), "Technical Specification Group Radio Access Network; Study on New Radio (NR) access technology (Rel. 14)," June 2017.

[28] V. Raghavan, A. Partyka, S. Subramanian, A. Sampath, O. H. Koymen, K. Ravid, J. Cezanne, K. K. Mukkavilli, and J. Li, "Millimeter wave MIMO prototype: Measurements and experimental results," IEEE Commun. Magaz., vol. 56, no. 1, pp. 202-209, Jan. 2018.

[29] V. Raghavan, L. Akhoondzadeh-Asl, V. Podshivalov, J. Hulten, M. A. Tassoudji, O. H. Koymen, A. Sampath, and J. Li, "Statistical blockage modeling and robustness of beamforming in millimeter wave systems," Submitted to IEEE Trans. Microwave Theory and Tech., Oct. 2018, Available: [Online]. https://arxiv.org/abs/1801.03346.

[30] V. Raghavan, V. Podshivalov, J. Hulten, M. A. Tassoudji, A. Sampath, O. H. Koymen, and J. Li, "Spatio-temporal impact of hand and body blockage for millimeter-wave user equipment design," IEEE Commun. Magaz., vol. 56, no. 12, pp. 46-52, Dec. 2018.

[31] Sony and Ericsson, "R4-1800889, Impact of UE back cover material on peak EIRP at mmWave," 3GPP TSG-RAN WG4 Meeting AH-1801, San Diego, CA, Jan. 2018.

[32] Qualcomm, "R4-1800369, On UE packaging loss, Simulation results,' 3GPP TSG-RAN WG4 Meeting AH-1801, San Diego, CA, Jan. 2018.

[33] Qualcomm, "Press release, July 23, 2018," Available: [Online]. https://www.qualcomm.com/news/releases/2018/07/23/ qualcomm-delivers-breakthrough-5g-nr-mmwave-and-sub-6 -ghz-rf-modules-mobile.

[34] V. Raghavan, J. Cezanne, S. Subramanian, A. Sampath, and O. H. Koymen, "Beamforming tradeoffs for initial UE discovery in millimeter-wave MIMO systems," IEEE Journ. Sel. Topics in Sig. Proc., vol. 10, no. 3, pp. 543-559, Apr. 2016.

[35] Sony and Ericsson, "R4-1704866, Frequency response of UE mmWave antennas for the $28 \mathrm{GHz}$ band," 3GPP TSG-RAN WG4 Meeting \#83, Hangzhou, China, June 2017.

[36] K. Sahota (Qualcomm), "5G mmWave radio design for mobile," IEEE 5 G summit, Honolulu, HI, June 2017, Available: [Online]. http://5gsummit.org/hawaii/docs/slides/ D2_\%239_Sahota_5g_060617.pdf.

[37] W. Hong, K-H. Baek, and S. Ko, "Millimeter-wave 5G antennas for smartphones: Overview and experimental demonstration," IEEE Trans. Ant. Propagat., vol. 65, no. 12, pp. 6250-6261, Dec. 2017.

[38] W. Hong, Z. H. Jiang, C. Yu, J. Zhou, P. Chen, Z. Yu, H. Zhang, B. Yang, X. Pang, M. Jiang, Y. Cheng, M. K. T. Al-Nuaimi, Y. Zhang, J. Chen, and S. He, "Multibeam antenna technologies for $5 \mathrm{G}$ wireless communications," IEEE Trans. Ant. Propagat., vol. 65, no. 12, pp. 6231-6249, Dec. 2017.
[39] Ericsson and Sony, "R4-1609590, UE reference architecture for NR," 3GPP TSG-RAN WG4 Meeting \#81, Reno, NV, Nov. 2016.

[40] J. Helander, K. Zhao, Z. Ying, and D. Sjöberg, "Performance analysis of millimeter-wave phased array antennas in cellular handsets," IEEE Ant. and Propagat. Letters, vol. 15, pp. 504-507, 2016.

[41] J. Zhang, X. Ge, Q. Li, M. Guizani, and Y. Zhang, "5G millimeterwave antenna array: Design and challenges," IEEE Wireless Commun., vol. 24, no. 2, pp. 106-112, Apr. 2017.

[42] L. Zhu and J. Zhu, "Optimal design of uniform circular antenna array in mmWave LOS MIMO channel," IEEE Access, vol. 6, pp. 61022-61029, 2018

[43] C. A. Balanis, Antenna Theory: Analysis and Design, WileyInterscience, 3rd edition, 2005.

[44] T. K. Y. Lo, "Maximum ratio transmission," IEEE Trans. Commun., vol. 47, no. 10, pp. $1458-1461$, Oct. 1999.

[45] X. Lin, J. Li, R. Baldemair, T. Cheng, S. Parkvall, D. Larsson, H. Koorapaty, M. Frenne, S. Falahati, A. Grövlen, and K. Werner, "5G New Radio: Unveiling the essentials of the next generation wireless access technology," 2018, Available: [Online]. https://arxiv.org/pdf/1806.06898. 